\begin{tabular}{c} 
International Journal of Engineering \& Technology, $7(3.33)(2018) 54-57$ \\
International Journal of Engineering \& Technology \\
Website: $w$ ww.sciencepubco.com/index.php/IJET \\
Research paper \\
\hline
\end{tabular}

\title{
Network Quality Measurements of LTE Drones
}

\author{
JeongWoo Jwa ${ }^{1}$, JoongHie Won ${ }^{2}$ \\ ${ }^{1}$ Department of Telecommunication Eng., Jeju National University, \\ Jeju Special Self-Governing Province, 63243, Republic of Korea \\ ${ }^{2}$ VICS, 815, Daewangpangyo-ro, Sujeong-gu, \\ Seongnam-si, Gyeonggi-do, KOREA Republic of Korea \\ *Corresponding author E-mail: E-mail: lcr02@jejunu.ac.kr
}

\begin{abstract}
Cellular-connected drones can provide beyond line-of sight control (BVLOS), low latency, real time communication, robust security, and wide range coverage. Cellular-connected drones have several key potential applications in enhanced LTE and 5G networks. The objective of the study is to evaluate field measurement data collected during drone flights in the KT Long-Term Evolution (LTE) networks in Seoul and Jeju, Korea. The multicopter drones are developed with LTE modem and LTE test smart phone for field measurements. The radio network quality between drones and LTE base transceiver stations (BTS) is evaluated by measuring downlink metrics such as the serving cell RSRP, RSRQ, SINR, modulation types of downlink, the downlink and uplink throughputs, and LTE PUSCH Tx Power on reference signal. In the existing LTE networks, field measurements show that there is no problem in C\&C of BVLOS operations in the flight height up to $150 \mathrm{~m}$ but the data rate requirements for video transmission does not meet at high flight altitudes.
\end{abstract}

Keywords: UAV, Drone, cellular network, LTE, reliable communication.

\section{Introduction}

Drones are often guided by remote controls by human pilots or they fly autonomously according to the predefined paths [1]. Drones are originally built for military purposes, but now the commercial drones market is rapidly growing. Drones are used in several fields such as photography and filming, infrastructure inspections and monitoring, precision agriculture, package delivery and disaster-relief applications, surveillance and monitoring, telecommunications, delivery of medical supplies, and rescue operations [2]. Drones have become important solutions considered among big retailers for deliveries. Amazon plans to use commercial drones to deliver goods [3]. Google's Project Wing tests a food delivery system for 30 minute delivery at Virginia Tech [4].

Current regulations in most countries limit drone operations and the operating ranges are termed either VLOS (visual line-of-sight) or BVLOS (beyond VLOS) operations between drones and their pilots. The distance controlled by the RF system is very short as the drone must be controlled within the visible range. Drones cannot fly higher than 150 meters (492 feet) when flying in VLOS operations according to South Korea drone laws. South Korea will allow drones to fly at night and deliver packages up to $5.5 \mathrm{~km}$ to support the fast-growing drone industry when pilots meet safety requirements. Advanced drones consist of camera, WiFi including high gain directional antenna, and LTE (Long-Term Evolution) modem. Development in flight control algorithms, machine vision, and onboard processing power will further enable these drones to take decisions themselves rather than relying on humans with less maneuvering expertise. Drones should also be equipped with an anti-collision system and a global positioning system transmitter that helps track their locations in case of crashes. Drones should also be equipped with an infrared camera and FPV (first-person view) devices. FPV is used as an essential device to provide the long-range mission of industrial drones. BVLOS operations will be allowed for extended flight range using a reliable $\mathrm{C} 2$ (command and control) link to the drone. Cellular networks are an attractive solution to provide the $\mathrm{C} 2$ connectivity.

The existing LTE networks can support VLOS as well as BVLOS operations of low altitude drones. The distinct features of various drones such as the maximum operational altitude, flying distance and coverage, data rate, QoS for drones can be considered in the future cellular networks. LTE with down tilted BTS's antenna optimizes the terrestrial coverage of the ground users in the cellular networks. Therefore, the quality of radio communication networks between drones and BTSs (base transceiver stations) is considerably different from that observed by the ground level. The air-to-ground propagation channel model for drones can be modeled as the free-space propagation model [5][6] at high flight heights. Enhanced LTE support for aerial vehicles in the 3GPP technical report [7][8] includes the radio propagation channel model and coverage and capacity provided by cellular networks to UAV's connectivity. The report shows that $5 \mathrm{G}$ (fifth generation) networks will provide more efficient connectivity for wide-scale drone deployments. Cellular-connected drones will be the most important use cases in 5G mobile networks [9][10]. In this paper, the downlink performance between BTS and drones is evaluated to provide BVLOS operations of low altitude drones.

\section{LTE Network Quality for LTE Drones}

\subsection{LTE Radio Network Quality Parameters}

Requirements for aerial vehicles agreed in 3GPP as follows: (1) data types are command and control $(\mathrm{C} \& \mathrm{C})$ and application data 
including video (streaming), images, other sensor data, etc., (2) heights target is up to $300 \mathrm{~m}$ above ground level, (3) speeds target horizontal speeds is up to $160 \mathrm{~km} / \mathrm{h}$, (4) latency is less than $50 \mathrm{~ms}$ (one way from evolved NodeBs to UAV), (5) data rates of C\&C are 60 100 kbps for uplink and downlink and application data is up to $50 \mathrm{Mbps}$ for UL (6) C\&C reliability of PER (packet error rate) is as low as $10^{-3}$.

In an LTE network, the UE (user equipment) measures downlink metrics on reference signal such as the RSRP (reference signal received power) and RSRQ (reference signal received quality) to select the serving cell and handoff. SINR (signal to interferenceplus-noise ratio), RSSI (received signal strength indicator) are measured to estimate of the signal quality at the UE. RSRQ and SINR are key metrics for LTE systems. UE measures SINR on each RB that can be defined as the ratio of the signal power to the summation of the average interference power from the other cells and the background noise. CQI (channel quality indicator) indicates the highest modulation and the code rate to show the communication channel quality of LTE. UE converts SINR to CQI and reports it to eNodeB. The CQI value ranges from 1 to 15 (15: the best channel quality, 1: the poorest channel quality, 0 : out of range).

RSRP and RSRQ are key measurement parameters indicating the received power level and the received signal quality level, respectively. RSRP is the average power of REs (resource elements) that carry CSRSs (cell specific reference signals) and RSSI measures all the power including all interference and noise over the entire bandwidth. The reporting range of RSRP is defined from -140 $\mathrm{dBm}$ to $-44 \mathrm{dBm}$. RSRQ includes the effect of interference from neighbor cells and is calculated from the RSRP and RSSI as follows:

\section{$R S R Q=\left(N^{*} R S R P\right) / R S S I$}

where $\mathrm{N}$ is the amount of RBs (resource blocks) which depends on the channel bandwidth. The reporting range of RSRQ is defined from $-19.5 \mathrm{~dB}$ to $-3 \mathrm{~dB}$. The RSRQ measurement provides additional information to make a reliable handover or cell re-selection decision.

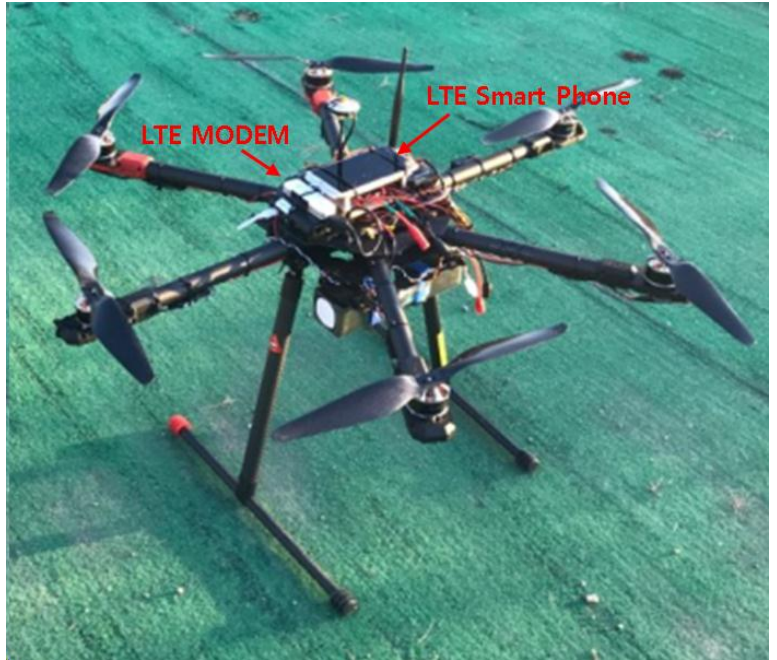

Fig. 1: The multicopter drone developed for filed measurements of LTE radionetwork quality

\subsection{Field Measurements of LTE Radio Network Quality}

In LTE networks, the BTS's antennas are down-tilted to optimize the terrestrial coverage and to provide good service for terrestrial UEs. We investigate the quality of the link between BS and UAVUEs to provide BVLOS operations of low altitude drones. We perform field measurements of the KT LTE radio network of $20 \mathrm{MHz}$ bandwidth in $1.8 \mathrm{GHz}$ band in urban and suburban areas in Seoul and Jeju, Korea, respectively. We use the KT radio field measurement and analysis tool of VoiceSniper ${ }^{\mathrm{TM}}$ and the LG LTE test phone. We use the developed multicopter with a LTE modem and the LG LTE smart phone as shown in Fig. 1. We perform drone flights in the predetermined route to measure LTE network quality according to drone flight altitude. The field measurements were carried out for heights up to a legally permitted maximum altitude of $150 \mathrm{~m}$ in increments of $30 \mathrm{~m}$. We also perform the field measurements using android app on the LTE Samsung smart phone and DJI Phantom 4Pro.

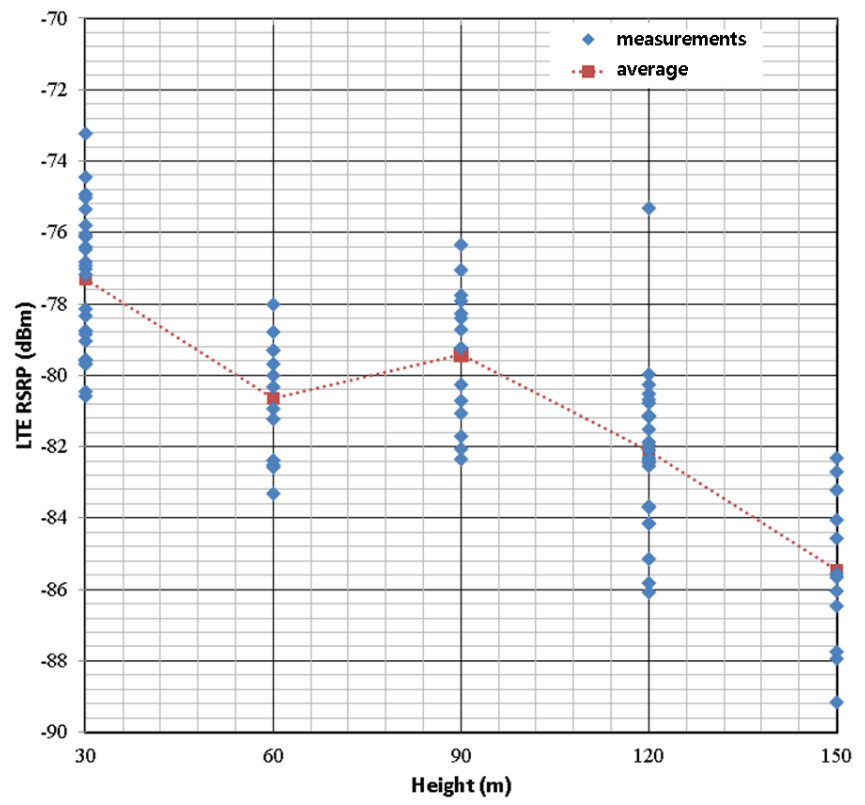

(a) RSRP

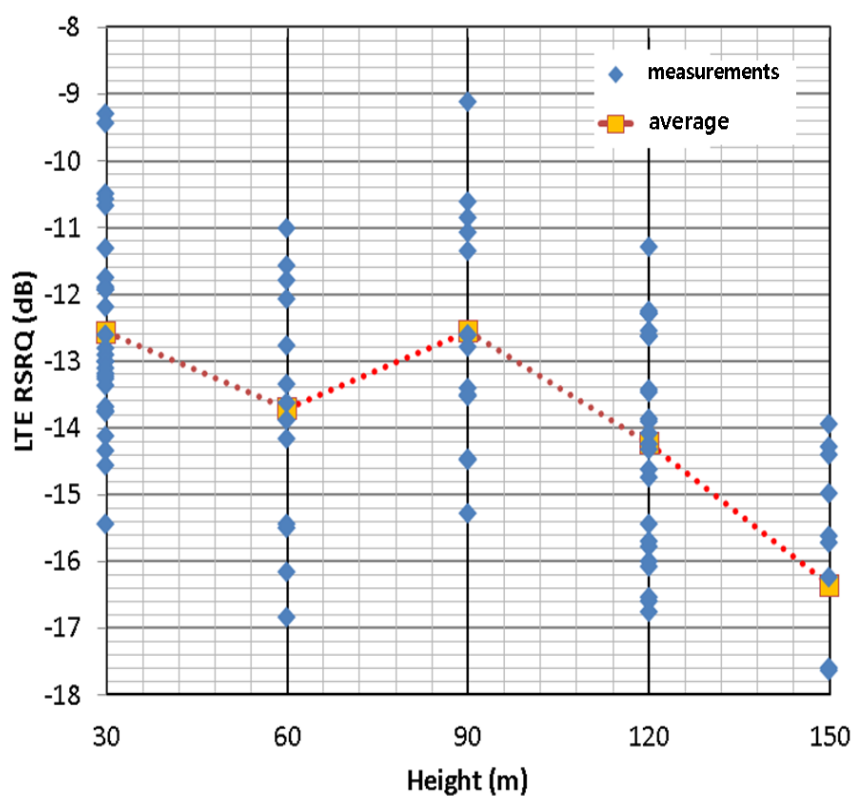

(b) RSRQ

Fig. 2: The distributions of the serving cell RSRP and RSRQ data according to flight altitudes

Figure 2 shows the distributions of the serving cell RSRP and RSRQ data. RSRP is a measurement parameter indicating the received power level in an LTE network. Field measurements show that the received power level is getting worse as flight altitude increases. The average RSRP data are $-72.30,-80.64,-79.42$, $-82.12,-85.45 \mathrm{dBm}$ at the heights of $30,60,90,120,150 \mathrm{~m}$, respectively. RSRQ is a measurement parameter indicating the received signal quality level. The radio propagation channel model can be represented as free-space propagation model at higher drone heights. The interference power from neighbor cells is in- 
creased as the number of neighbor cells observed is increased at higher drone heights. Therefore, the download interference power from neighbor cells degrades the downlink performance between BTS and drones at higher drone heights. Field measurements show that the cell selection/reselection and handover frequently occurs at higher altitude. The average RSRQ data are -12.56 , $13.71,-12.55,-14.24,-16.35 \mathrm{~dB}$ at the heights of $30,60,90,120$, $150 \mathrm{~m}$, respectively.

Figure 3 shows the distribution of the serving cell SINR and modulation types such as 64QAM, 16QAM, and QPSK. SINR of the received signal quality metric is converted to CQI and downlink modulation type is determined. Field measurements show that SINR also degrades as the flight altitude increases as that of RSRQ due to downlink interference from non-serving cells. The average SINR is $6.11,3.35,4.28,1.22,-1.68 \mathrm{~dB}$ at the heights of $30,60,90,120,150 \mathrm{~m}$, respectively. Figure $3(\mathrm{~b})$ shows that the percentage of 64 QAM is $26.34,16.58,27.87,18.98,8.98 \%$ at the heights of 30,60,90,120,150 m, respectively. At height of $150 \mathrm{~m}$, the percentage of 64QAM, 16QAM, and QPSK is 58.25, 32.54, $8.93 \%$, respectively. Field measurements show that the percentage of 64 QAM is decreased as the flight altitude increases.

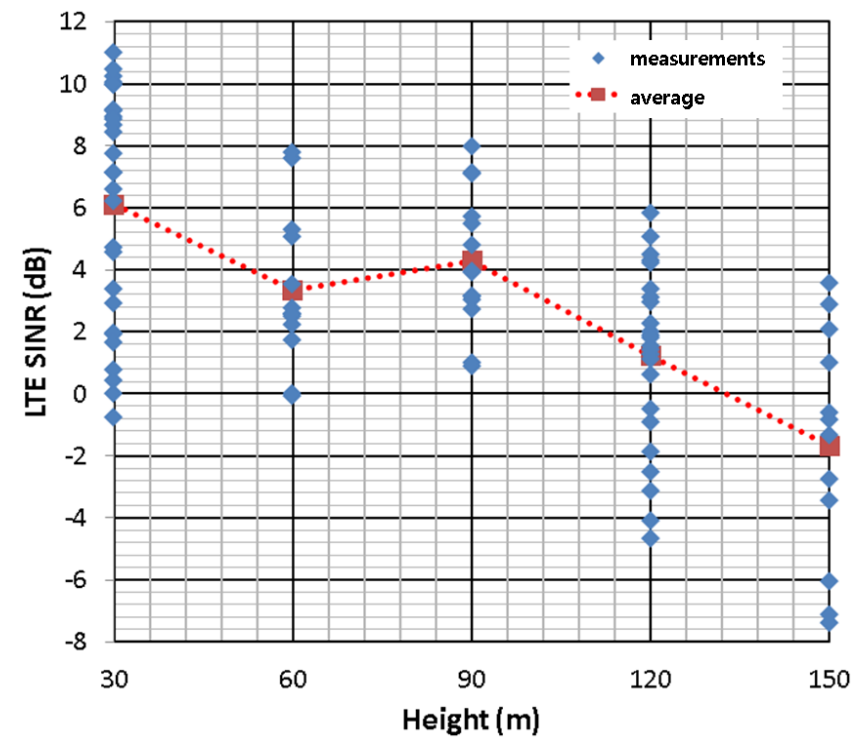

(a) SINR

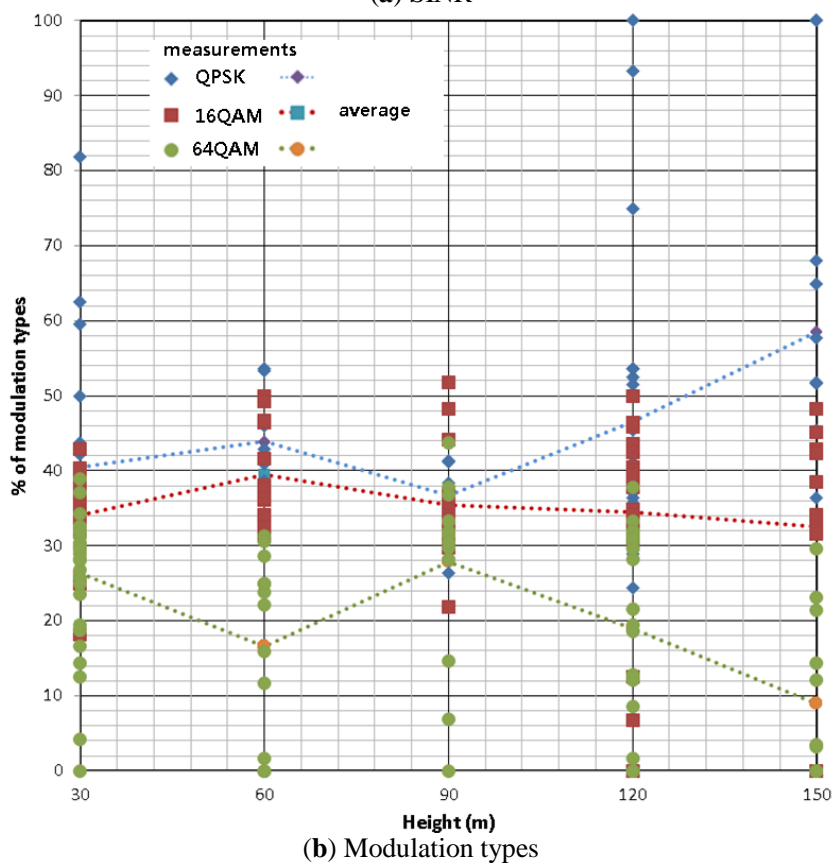

Fig. 3: The distributions of the serving cell SINR data and modulation types according to flight altitudes.
We measure throughputs of the downlink and uplink with file downloading and uploading. Figure 4 shows average data rate of uplink relative to flight altitudes. The average data rate of downlink is 53.70, 43.93, 49.76, 36.08, 28.14 Mbps at the heights of 30, $60,90,120,150 \mathrm{~m}$, respectively. From the field measurement results, we can see that the uplink data rate sometimes occurs below $30 \mathrm{Mbps}$ when the heights of the drone are over $120 \mathrm{~m}$. The existing LTE systems do not meet the data rate requirements for video transmission at high flight altitudes. The average data rate of uplink is the range of $18 \sim 19 \mathrm{Mbps}$ regardless of the height of the drones. There is no problem in transmitting $\mathrm{C} \& \mathrm{C}$ data of the requirement of $60 \sim 100 \mathrm{kbps}$ on the uplink in the flight height up to $150 \mathrm{~m}$.

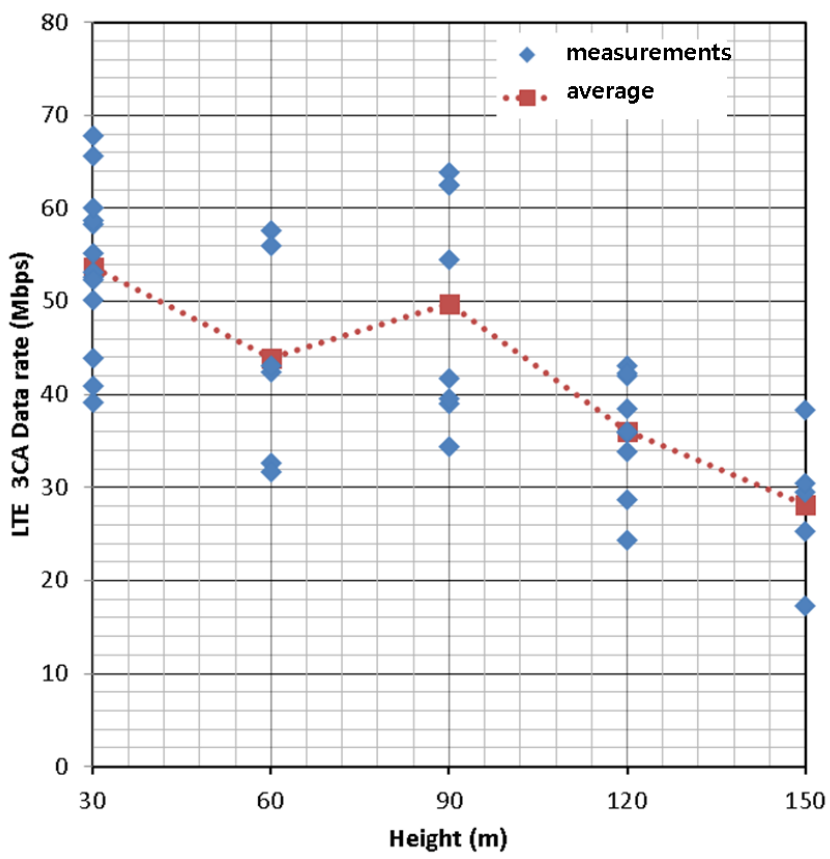

Fig. 4: The distributions of the serving downlink data rate according to flight altitudes.

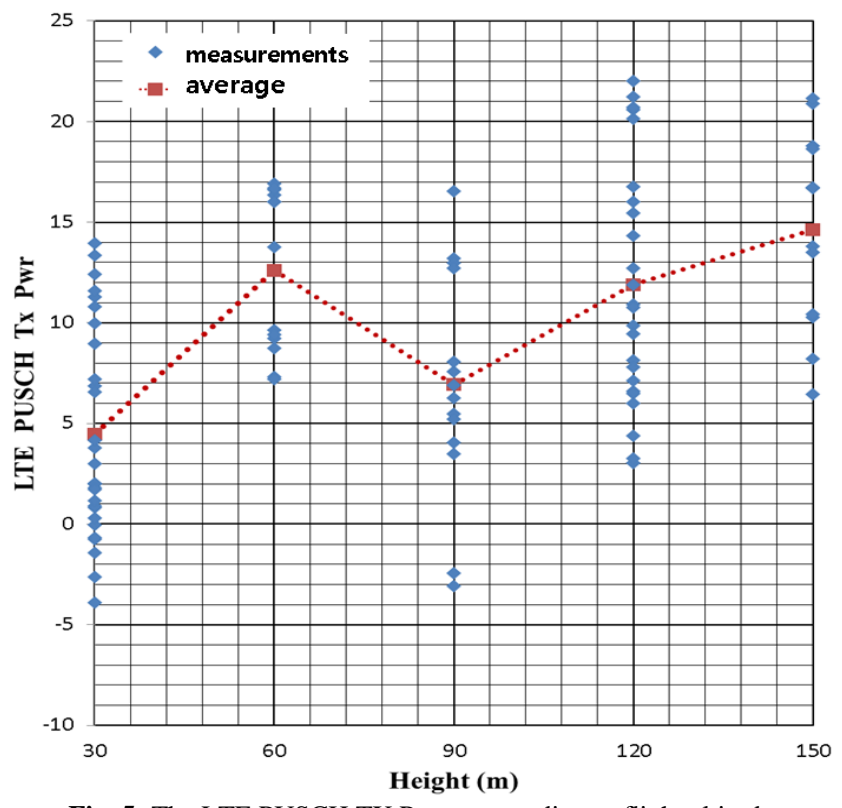

Fig. 5: The LTE PUSCH TX Power according to flight altitudes

We measure the PUSCH (Physical Uplink Shared Channel) Tx (transmit) power relative to flight altitudes as shown in Fig. 5. The average values of the LTE PUSCH Tx Power are 4.47, 12.61, 6.92, $11.90,14.63 \mathrm{dBm}$ at the heights of $30,60,90,120,150 \mathrm{~m}$, respectively. 


\section{Conclusion}

The existing LTE networks can support BVLOS operations of low altitude drones. Enhanced LTE and 5G networks can support the reliable command and control $(\mathrm{C} \& \mathrm{C})$ as well as the reliable application data including video (streaming), images, other sensor data, etc. Enhanced LTE and 5G networks can provide heights target up to $300 \mathrm{~m}$ and data rates for $\mathrm{C} \& \mathrm{C}$ up to $60 \sim 100 \mathrm{kbps}$ and application data up to $50 \mathrm{Mbps}$ for UL. The objective of the study is to evaluate the radio network quality between drones and LTE BTS by measuring downlink metrics such as the serving cell RSRP, RSRQ, SINR on reference signal. The modulation types of downlink, CQI, PCIs (physical cell identifiers), the downlink and uplink data rate, and LTE PUSCH Tx Power are also measured to to evaluate the radio network quality. Field measurements show that the radio network quality degrades at the heights higher than $120 \mathrm{~m}$ as the effect of substantial interference from neighbor cells. The numbers of observed neighbor cells are increased as the radio path clearance at the higher flight heights. Field measurements show that there is no problem in C\&C of BVLOS operations in the existing LTE networks in the flight height up to $150 \mathrm{~m}$ but the data rate requirements for video transmission does not meet at high flight altitudes. In enhanced LTE and 5G networks, interference cancellation and beam forming antenna techniques can be used for cellular-connected drones.

\section{Acknowledgement}

This research was supported by Basic Science Research Program through the National Research Foundation of Korea (NRF) funded by the Ministry of Education (2017036515). This work (Grants No.0514401) was supported by Business for Cooperative R\&D between Industry, Academy, and Research Institute funded Korea Small and Medium Business Administration in 2017.

\section{References}

[1] F. Kendoul, "Survey of advances in guidance, navigation, and control of unmanned rotorcraft systems," J. Field Robot., vol. 29, no. 2 (2012), pp. 315-378

[2] P.E. Ross, "Top 11 technologies of the decade," IEEE Spectrum, vol. 48, no. 1(2011), pp. 27-63.

[3] Amazon, "Amazon prime air," https://www.amazon.com/ b? node $=8037720011$

[4] M. McFarland, "Google drones will deliver chipotle burritos at Virginia Tech,” CNN Money (2016).

[5] H. C. Nguyen, R. Amorim, J. Wigard, I. Z. Kovcs, T. B. Srensen, and P. Mogensen, "How to ensure reliable connectivity for aerial vehicles over cellular networks," IEEE Access, vol. 6 (2018), pp. 12304-12317.

[6] X. Lin, R. Wiren, S. Euler, A. Sadam, H.-L. Maattanen, S. D Muruganathan, S. Gao, Y.-P. E. Wang, J. Kauppi, Z. Zou, and V. Yajnanarayana, "Mobile networks connected drones: Field trials, simulations, and design Insights," submitted to IEEE Communications Magazine(2018). Available at http://arxiv.org/abs/1801.10508

[7] Study on Enhanced LTE Support for Aerial Vehicles (Release 15), document 3GPP TR 36.777 V0.4.0 (2017-11), 3GPP, Technical Specification Group Radio Access Network, (2017).

[8] S. D. Muruganathan, X. Lin, Helka-Liina Maattanen, Z. Zou, W. A Hapsari, S. Yasukawa, "An Overview of 3GPP Release-15 Study on Enhanced LTE Support for Connected Drones" arXiv:1805.00826 (2018).

[9] Y. Zeng, R. Zhang, and T. J. Lim, "Wireless communications with unmanned aerial vehicles: Opportunities and challenges," IEEE Commun. Mag., vol. 54, no. 5(2016), pp. 36-42.

[10] I. Parvez, A. Rahmati, I. Guvenc, A. I. Sarwat, and H. Dai, "A Survey on Low Latency Towards 5G: RAN, Core Network and Caching Solutions," CoRR, vol. abs/1708.02562, 2017. [Online]. Available: http://arxiv.org/abs/1708.02562 (2017). 